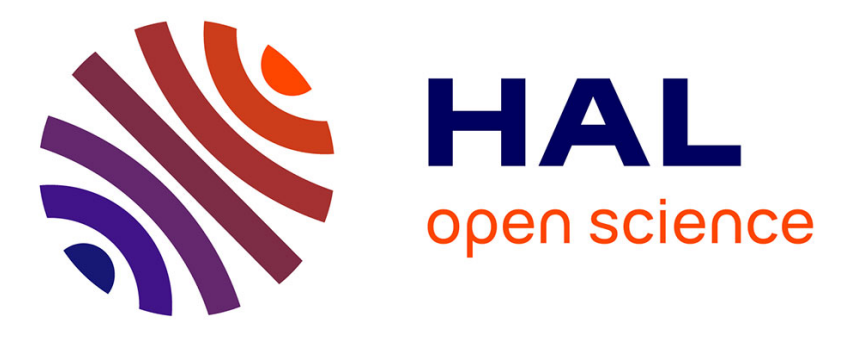

\title{
Coherent beam combining of 61 femtosecond fiber amplifiers
}

\author{
Ihsan Fsaifes, Louis Daniault, Severine Bellanger, Matthieu Veinhard, Jerome \\ Bourderionnet, Christian Larat, Eric Lallier, Eric Durand, Arnaud Brignon, \\ Jean-Christophe Chanteloup
}

\section{To cite this version:}

Ihsan Fsaifes, Louis Daniault, Severine Bellanger, Matthieu Veinhard, Jerome Bourderionnet, et al.. Coherent beam combining of 61 femtosecond fiber amplifiers. Optics Express, 2020, 28 (14), pp.20152.

10.1364/oe.394031 . hal-02995221

\section{HAL Id: hal-02995221 \\ https://hal.science/hal-02995221}

Submitted on 9 Nov 2020

HAL is a multi-disciplinary open access archive for the deposit and dissemination of scientific research documents, whether they are published or not. The documents may come from teaching and research institutions in France or abroad, or from public or private research centers.
L'archive ouverte pluridisciplinaire HAL, est destinée au dépôt et à la diffusion de documents scientifiques de niveau recherche, publiés ou non, émanant des établissements d'enseignement et de recherche français ou étrangers, des laboratoires publics ou privés. 


\title{
Coherent beam combining of 61 femtosecond fiber amplifiers
}

\author{
Ihsan Fsaifes, ${ }^{1,}{ }^{*}$ Louis Daniault, ${ }^{1}$ (D) SeVerine Bellanger, ${ }^{1}$ \\ MatthiEu Veinhard, ${ }^{1}$ Jerome Bourderionnet, ${ }^{2}$ Christian \\ LARAT, ${ }^{2}$ ERIC LALLIER, ${ }^{2}$ ERIC DURAND, ${ }^{3}$ ARnAUd BrIGNON, ${ }^{2}$ AND \\ JEAN-ChRISTOPHE ChANTELOUP ${ }^{1}$ \\ ${ }^{1}$ XCAN, Ecole Polytechnique, Institut Polytechnique de Paris, 91128 Palaiseau Cedex, France \\ ${ }^{2}$ Thales Research \& Technology, 1 avenue Augustin Fresnel, 91767 Palaiseau Cedex, France \\ ${ }^{3}$ Thales LAS France SAS, 2 avenue Gay Lussac, 78995 Elancourt Cedex, France \\ *ihsan.fsaifes@polytechnique.edu
}

\begin{abstract}
We report on the coherent beam combining of 61 femtosecond fiber chirped-pulse amplifiers in a tiled-aperture configuration along with an interferometric phase measurement technique. Relying on coherent beam recombination in the far field, this technique appears suitable for the combination of a large number of fiber amplifiers. The 61 output beams are stacked in a hexagonal arrangement and collimated through a high fill factor hexagonal micro-lens array. The residual phase error between two fibers is as low as $\lambda / 90$ RMS, while a combining efficiency of $\sim 50 \%$ is achieved.
\end{abstract}

(C) 2020 Optical Society of America under the terms of the OSA Open Access Publishing Agreement

\section{Introduction}

A wide field of applications ranging from materials processing [1-3] to high-field physics [4] requires amplification laser systems delivering both high average and peak powers at high repetition rate and efficiency. It is now becoming a reality thanks to tremendous recent progresses in high power ultrafast thin disk lasers [5] but also in a more scalable laser architectural approach called Coherent Beam Combining (CBC) [6,7] with the $\mathrm{kW}$ average power horizon already exceeded for both technologies. The principle of CBC is to divide a single source into $\mathrm{N}$ independent channels, each with a dedicated amplifier. The outputs of the $\mathrm{N}$ amplified channels are ultimately coherently combined into one single beam, which carries $\mathrm{N}$ times the power of a single channel. For this recombination to be efficient, the phases of the sub-beams need to be adjusted such that they match each other [8]. The amplifiers of choice for the vast majority of CBC laser prototypes currently studied or operated are fiber amplifiers. Such amplifiers carrie indeed advantageous characteristics such as excellent beam quality, high efficiency and good thermal handling.

$\mathrm{CBC}$ can be performed either in filled-aperture or in tiled-aperture configuration. In filledaperture, several beams are efficiently combined into a single beam by using polarization beam splitters [9], diffractive optical elements [10], or segmented mirror splitter/combiner [11].

The tiled aperture approach described in this work shall be considered as an experimental proof of concept demonstrating the scaling potential of a compact and robust high average/peak power CBC laser system. Compactness is ensured thanks to the use of flexible fibers for all amplifying stages and to the millimeter-scale fibers arrangement within a 2D-array laser head (with very stringent pitch and angular positioning accuracies requirements). Robustness is achieved through the use of a single focusing lens to achieve CBC.

Applications in the field of high-energy physics, such as particle acceleration $[12,13]$ and space debris removal $[14,15]$, would benefit from the high average powers provided by fiber laser systems, but would require pulse energies of up to $10 \mathrm{~J}$. Considering the milli-joule level of a 
single emitter, several thousands of fibers would then have to be combined. Thus, highly scalable $\mathrm{CBC}$ architectures along with an adequate phase measurement technique is needed.

The XCAN (Coherent Amplification Network) project is a collaboration between the Ecole Polytechnique and the Thales company aiming at experimentally exploring such laser architecture using a scalable $\mathrm{CBC}$ approach in the femtosecond regime. Our CBC prototype consists in coherently adding, in a tiled-aperture configuration, the output beams of 61 independent fiber amplifiers seeded by a common femtosecond source, stacked in a hexagonal arrangement and collimated by a high fill factor hexagonal microlens array [16]. The phase correction is performed using an interferometric measurement method with active phase control and beam combination in far field. This technique is suitable for the co-phasing of a large number of beams, since the recombination and the phase measurements are achieved in one single step.

Proofs of principle with prototypes of similar architectures have first been demonstrated with 19 passive fibers [17], and subsequently with 7 and 37 high power fiber amplifiers [16,18]. In this paper, we report on the $\mathrm{CBC}$ of 61 femtosecond fiber chirped-pulse amplifiers operating at moderate average power in the linear regime and give first results on high power regime.

\section{Laser head}

Coherent beam combining efficiency in tiled-aperture configuration is highly sensitive to the accuracy of the fiber array alignment [19]. Typically, a single micrometer error in lateral positioning or a single milliradian error in beam-to-beam pointing will affect the efficiency by about $1 \%$. In order to arrange the 61 output fibers accurately in a hexagonal array and to satisfy the stringent $\mathrm{CBC}$ geometrical constraints (pitch and beam-to-beam pointing), we use a simple hand-stacking approach. The main part of the laser head is a high precision $60^{\circ} \mathrm{V}$-shape mechanical holder, in which high accuracy ceramic sleeves of $3.2 \mathrm{~mm}$ outer diameter are simply stacked into the $\mathrm{V}$, automatically arranging themselves into a regular hexagonal array.

In order to insert the power amplifiers extremities in this structure, a high precision ceramic ferrule is mounted at the end of the fibers, right before the endcap, so that they can be easily slipped into the sleeves. It is worth noting that the fiber ending including the ceramic ferrule and the endcap is carefully designed to guarantee a repeatable, break-safe fiber insertion into the ceramic sleeves and a safe extraction for maintenance purposes. When adding the ferrule around the fiber, special care is taken to ensure that the fiber output mode is well centered with respect to the ferrule outer diameter. More details about the fiber ending design are given in reference [20].

The fiber amplifiers are manufactured in such a way that its polarization axis aligns itself parallel to the coiling plane. The polarization of each fiber could be further optimized by rotating it a few degrees to maximize the amplifier output signal. Thus, all the beams are polarized along the same common axis. Nevertheless, this self-alignment polarization mechanism is not totally perfect as small individual deviations of the polarization axis may occur and have to be taken into account. To address this issue, the composite beam is sent through a half-wave plate and a thin film polarizer. Moreover, as the exact polarization directions vary slightly among the 61 individual beams, the wave plate allows adjusting the average polarization such that the sum of the power losses of each channel is minimized.

To make sure the fiber longitudinal position is the same for all 61 channels, a second metallic plate is placed in contact with the $\mathrm{V}$-shape holder. This plate is drilled with holes distributed over a $3.2 \mathrm{~mm}$ pitch regular hexagonal array in such a way that this hole network is perfectly aligned with the sleeve arrangement of the V-shaped holder. The holes diameters are slightly larger than the endcaps $(1 \mathrm{~mm})$ while being smaller than the ferule $(3.2 \mathrm{~mm})$. Thus, this second plate plays two roles. It first allows to align all ferules in the same common plane (located at the output port of the sleeves). It also collects a part of the unabsorbed $976 \mathrm{~nm}$ pump power refracted through the endcaps lateral surface. 
Such laser head allows to handle accurately a set of 61 laser outputs carrying globally more than a $\mathrm{kW}$ of average power. Figure 1 shows a picture of the laser head containing the 61 fiber amplifiers arranged within 9 stacked breadboards. The 61 outputs form a fiber bundle, accurately inserted into the laser head (the hexagonal arrangement can clearly be observed on the picture) and collimated with a hexagonal microlens array with a focal length of $66 \mathrm{~mm}$ (left on the image). Inset in Fig. 1 displays the entrance side of the laser head.

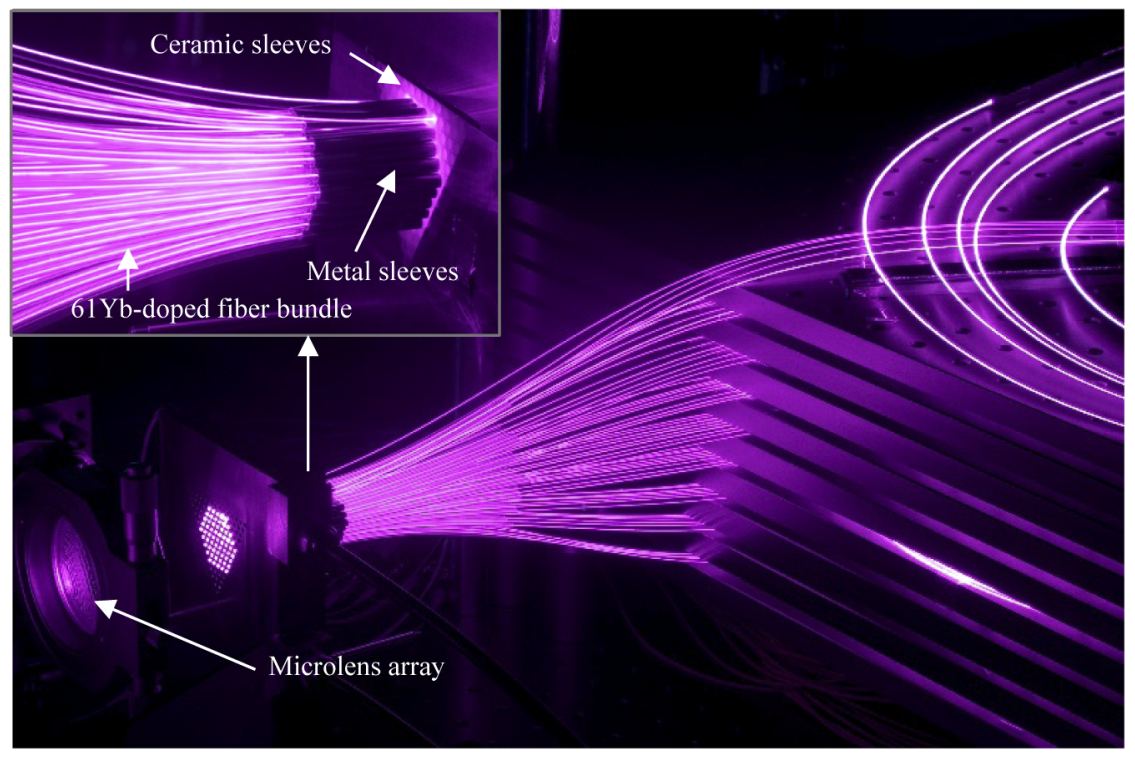

Fig. 1. XCAN laser head (center), microlens array (left) and 61 fiber bundle (right). Inset: entrance side of the laser head.

\section{Experimental setup and results}

The experimental setup is shown in Fig. 2. The master oscillator delivers $220 \mathrm{fs}$ laser pulses at a repetition rate of $55 \mathrm{MHz}$. The pulses are temporally stretched to $2.5 \mathrm{~ns}$ through a chirped fiber Bragg grating (C-FBG), followed by a pulse shaper common to all channels and used to pre-compensate the dispersion accumulated all over the chain. Three Acousto-Optic Modulators (AOM) are inserted into the laser chain as pulse-pickers to reduce the pulse repetition rate, consequently allowing to increase the output pulse energy and therefore operating XCAN in non-linear regime when requested.

The beam is then split into eight sub-beams, each one being again split into new eight sub-beams, 61 of them passing through individual Delay and Phase Adjustment Modules (DPAM) allowing independent adjustment of each individual optical path lengths. This ensures an exact match of phase offsets $\left(\varphi_{0}\right)$ and delays $\left(\varphi_{1}\right)$ between all the pulses as defined in equation (2) of [16], thus leading to a stable and efficient coherent combination. Delays are adjusted with motorized free space Variable Optical Delay Lines (VODL). Phase offsets control is performed while mechanically stretching a $15 \mathrm{~m}$ long fiber coiled around a piezo-ceramic cylinder (Fiber Stretcher, FS) with a driving voltage of $\pm 10 \mathrm{~V}$ (a phase-shift coefficient of $\sim 12 \mathrm{rad} / \mathrm{Volt}$ @ $1 \mu \mathrm{m}$ ) allowing a dynamic range of $\pm 19 \mu \mathrm{m}$, corresponding to phase shifts of $\pm 18,4 \lambda$. In order to compensate for power losses accumulated all over the laser chain, several pre-amplifiers delivering $100 \mathrm{~mW}$ of saturated output power are used.

The 61 beams are power amplified using Large Mode Area (LMA) Ytterbium-doped Photonic Crystal Fibers of $30 \mu \mathrm{m}$ mode field diameter, supplied by NKT Photonics and functionalized 


\section{Optics EXPRESS}

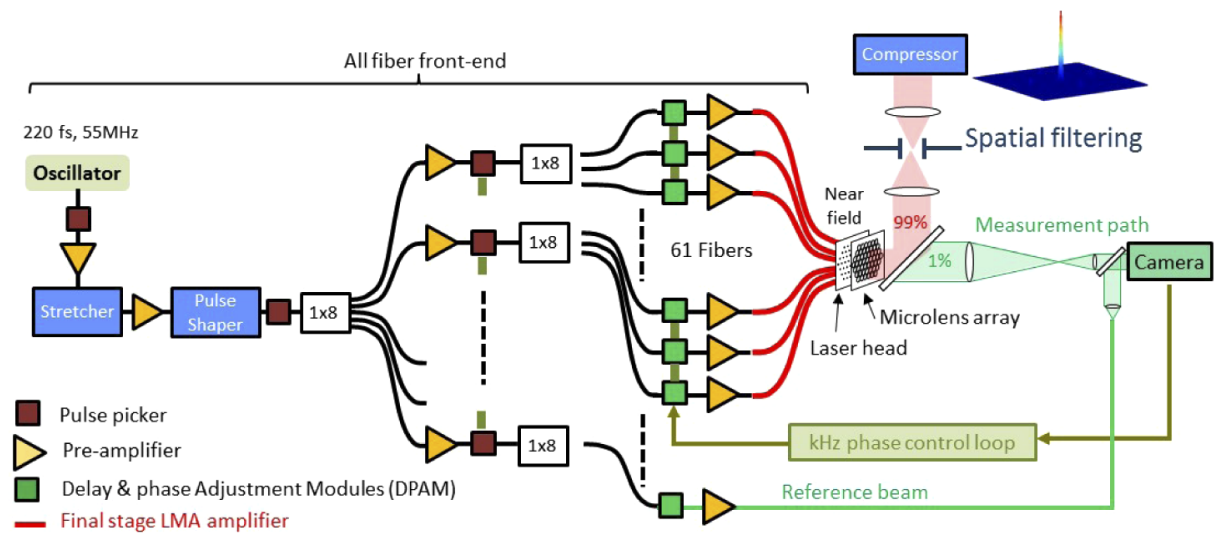

Fig. 2. Experimental setup for the $\mathrm{CBC}$ of 61 fiber amplifiers.

by Optical Engines Inc. A co-propagative pumping scheme was selected due to the laser head compactness (3.2 $\mathrm{mm}$ pitch) requirements described in section 2. Pump and signal are injected into the fiber amplifier along the same direction, through an all-fiber pump/signal combiner located before the active fiber.

Except for the pulse shaper and the free space VODLs, our laser system operates with an all-polarization maintaining fiber front-end, thus guaranteeing a maximal robustness and convenience of use. Considering the front-end and the active fiber amplifier (see Fig. 2), the laser amounts about $100 \mathrm{~m}$ of fiber per channel. Let's recall here that the fiber lengths of all the channels are matched to a few $\mathrm{cm}$ to fit into the VODLs free space range of $6 \mathrm{~cm}( \pm 3 \mathrm{~cm}$ with $0.1 \mu \mathrm{m}$ increment). This ensures that there are no CBC efficiency losses due to group velocity dispersion or higher order discrepancies.

CBC is performed by using the interferometric phase measurement method described in [8]. The phase measurement and correction is based on correlations between the camera signal (S) and pre-defined/pre-recorded reference patterns $(\mathrm{R})$. For a given image, the precision of the phase measurement depends on the calculated reference patterns number, the size of the camera window used to calculate the correlations and the number of pixels per fringe.

A small power fraction of the composite near field is imaged onto a $1 \mathrm{kfps}$ high speed $1280 \times 1024$ pixels $/ 14 \times 14 \mu \mathrm{m}^{2}$ pixel size camera, where it interferes with an expanded passive reference beam for real-time phase measurement. This allows the acquisition of 61 interferograms on a single camera image $(400 \times 400$ pixels recording pupil is sufficient as depicted on Fig. 3$)$, and the subsequent calculation of the relative phases between each channel and the reference beam [8].

A $1 \mathrm{kHz}$ feedback algorithm implemented on a Field-Programmable Gate Array (FPGA) acting on the DPAMs, actively cancels all these phase mismatches. However, a constant phase relationship remains between the fibers due to the geometric fiber alignment in the hexagonal array. In order to address it, a Stochastic Parallel Gradient Descent (SPGD) algorithm is used. The SPGD is a hill-climbing algorithm [7,21] used in our setup to finely co-phase the 61 individual fiber amplifiers with respect to each other by applying appropriate sets of small dithers to the 61 phase modulators. It allows to apply a correction of the static phase offsets of the individual beams mainly caused by the geometrical fibers distribution in the laser head (the constant phase relationships between the fibers) and some inconsistencies from the measurement paths (see Fig. 2). 


\section{Optics EXPRESS}
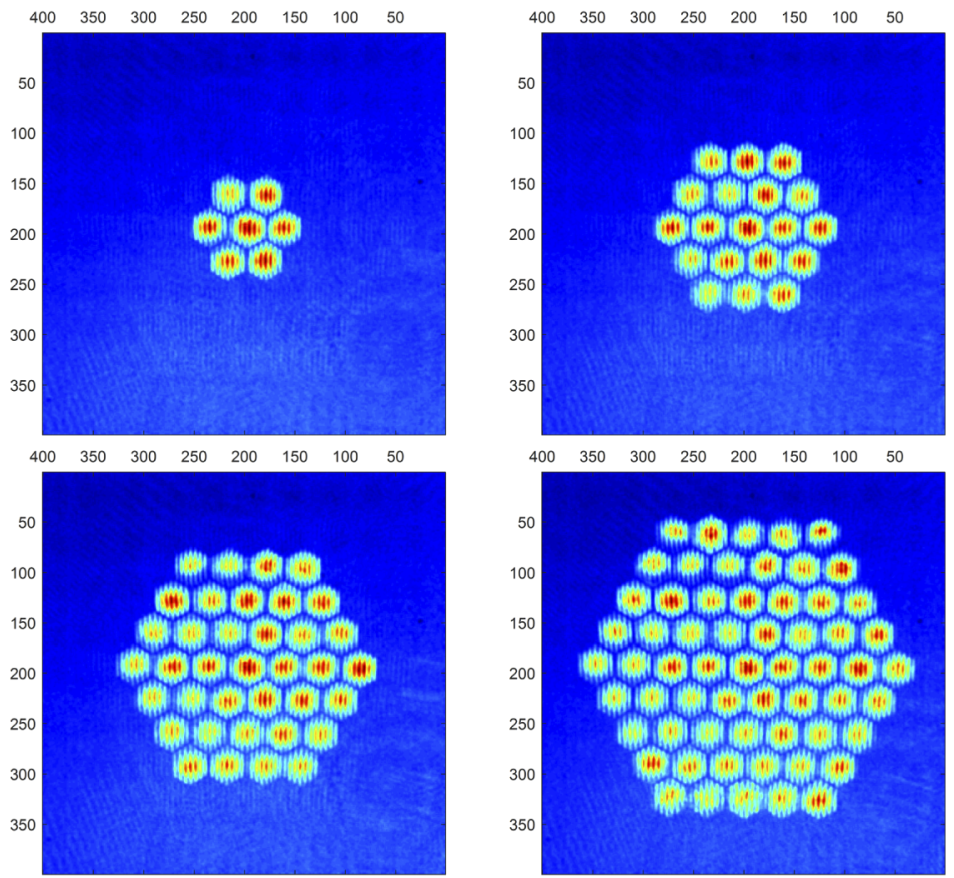

Fig. 3. Composite near field interference patterns of 7, 19, 37 and 61 fibers obtained with the expanded reference fiber beam. Beam to beam contrast variations are related to channels power variations.

In this way, and once the overall laser system is thermalized, the far-field main lobe (see Fig. 4) power is maximized, improving thus the combining efficiency. A simulated annealing approach can be also implemented whenever requested by the operational changes.
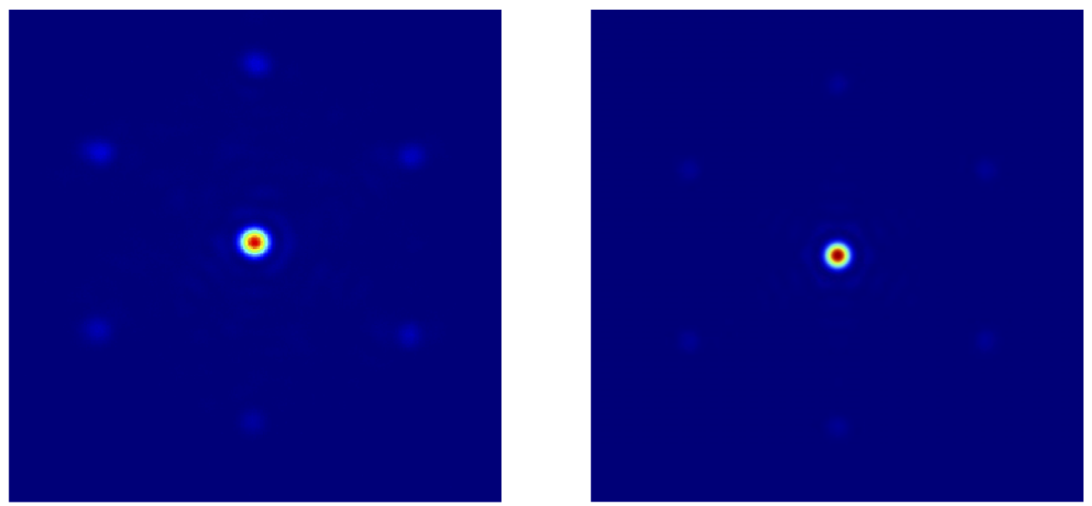

Fig. 4. Simulated (right) and experimental far field (left) obtained with the $\mathrm{CBC}$ of 61 beams in the linear regime with a total average power of $10 \mathrm{~W}(5.2 \mathrm{~W}$ in the main lobe). (see Visualization 1).

Figure 3 shows the near field interference patterns of 7, 19,37 and 61 fibers, illustrating the high scalability of the XCAN laser. Compared to a perfect hexagonal paving, a measurement of the residual errors at the laser head level yields to the following standard deviations: (1): 3.0 and $4.8 \mu \mathrm{m}$ on the microlens positioning along both transverse axis and (2): 2.7 and $3.4 \mathrm{mrad}$ for the 
beam-to-beam pointing. Despite these minimal residual misalignments, these patterns exhibit well-centered beams on the microlenses axes, demonstrating a good beam-to-beam pointing accuracy. Moreover, equal fringe periods and orientations attest nice fiber positioning along the transverse axes.

Figure 4 displays the experimental far field obtained with 61 amplified and collimated beams (left) and the corresponding simulated one (right) when the fiber array is phase-locked. Excellent agreement can be observed between the experimental and simulated far fields.

The periodic intensity distribution of the composite beam in the near field gives rise to six side-lobes with equalized intensities surrounding the main lobe as observed on these far fields. Thus, the combined beam corresponds only to the main lobe of the far field pattern, and the combining efficiency $\eta$ is defined as the average power in this main lobe divided by the overall average power in the far field. Finally, to obtain the effective combining efficiency, $\eta$ should be multiplied to the microlens transmission $t_{\mu-\text { lens }}$.

It is worth noting that the side-lobes set an upper limit of $67 \%$ to the combining efficiency, obtained for an optimum microlens fill factor of 0.93 (see Fig. 1 of [16]). A video file, available in Visualization 1 shows the time-varying speckle pattern when the control loop is open; turning into a stable far field pattern when the control loop is enabled.

The far field is then spatially filtered by truncating it at the first extinction of the main lobe. We measure a combining efficiency of 52\%. Taking into account the microlens transmission $t_{\mu-\text { lens }}=92 \%$ (microlenses clipping losses), the final effective combination efficiency is $48 \%$. This corresponds to $72 \%$ of the theoretical CBC efficiency of $67 \%$, considering a perfect fiber array. The remaining difference between the experimental and the theoretical efficiencies is due to some misalignments in the fiber array and polarization orientation errors.

It's worth noting that such efficiency level reveals that spectral phase discrepancies between the 61 beams have a minor effect on the CBC combination efficiency. As shown by Fig. 5, nearly identical spectra are obtained, in the linear regime $(55 \mathrm{MHz}$ repetition rate) for one channel (central fiber on the hexagonal array) and for the 61-combined beam (total average power in the main lobe of $\sim 5 \mathrm{~W}$ ).

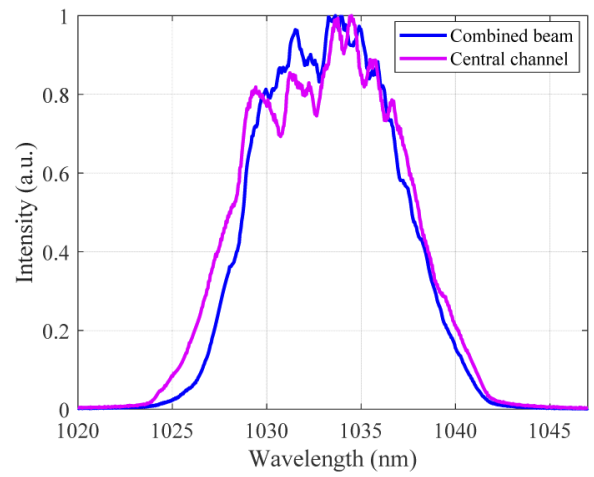

Fig. 5. Normalized spectra of one channel and the 61-combined beam.

The phase locking process was evaluated in the linear regime (low B-integral) at a repetition rate of $55 \mathrm{MHz}$ by measuring the phase noise between two fiber amplifiers operating in linear regime. The relative phase is retrieved by generating interference fringes between the two beams and simultaneously recording the interference states in quadrature using two independent photodiodes [7,8]. Figure 6 gives the phase noise spectral density of the measured relative phases in open and closed loops, respectively when the amplified signal power is set to $4 \mathrm{~W} / \mathrm{fiber}$ (left) and $20 \mathrm{~W} /$ fiber (right). 


\section{Optics EXPRESS}
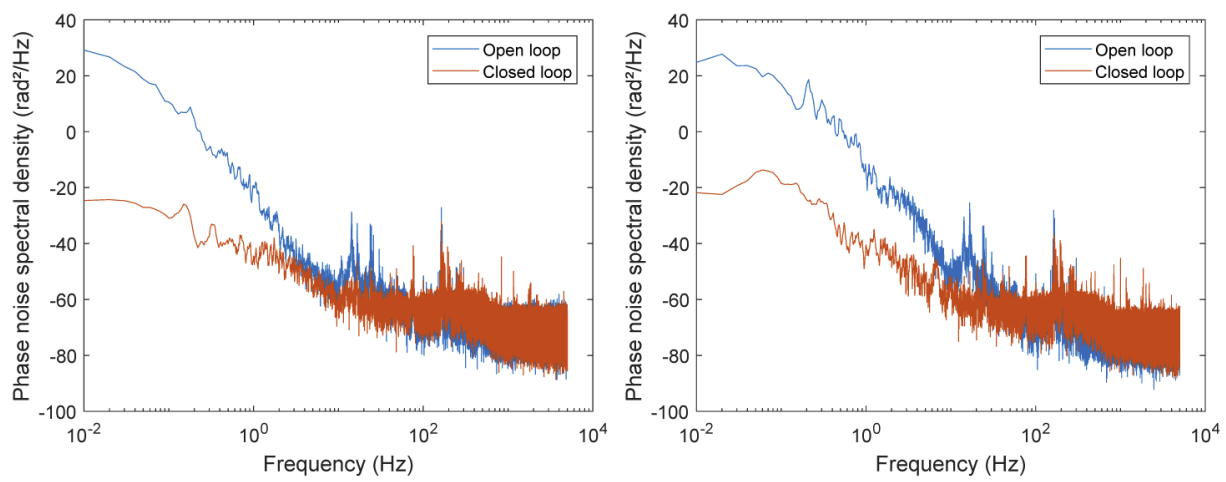

Fig. 6. Phase noise spectral density for open and closed loops. left: at low signal power/ fiber, right: at high signal power/ fiber.

In both cases, the feedback algorithm clearly reduces the low-frequency noise by more than four orders of magnitude in the low frequency band, yielding a residual phase error between two fibers of $\lambda / 93$ and $\lambda / 55$ RMS at low and high signal power respectively. Let's recall here that to calculate the residual phase error, we integrate the PSD curve (in $\mathrm{rad}^{2} / \mathrm{Hz}$ ) derived from the temporal signal to retrieve the variance, and thus the residual phase error with a square root.

The beam quality factor $\mathrm{M}^{2}$ was determined by focusing the 61 -combined beam and measuring the beam width as a function of longitudinal distance. Figure 7 displays the second-moment values of the intensity profiles for different propagation distances in the vicinity of the far field image. An $\mathrm{M}^{2}$ of 1.11 along the $\mathrm{x}$-axis and $\mathrm{M}^{2}$ of 1.03 along the $\mathrm{y}$-axis were obtained. Such high spatial beam quality confirms that the filtered $61-\mathrm{CBC}$ beam has a rather similar behavior as a Gaussian beam.
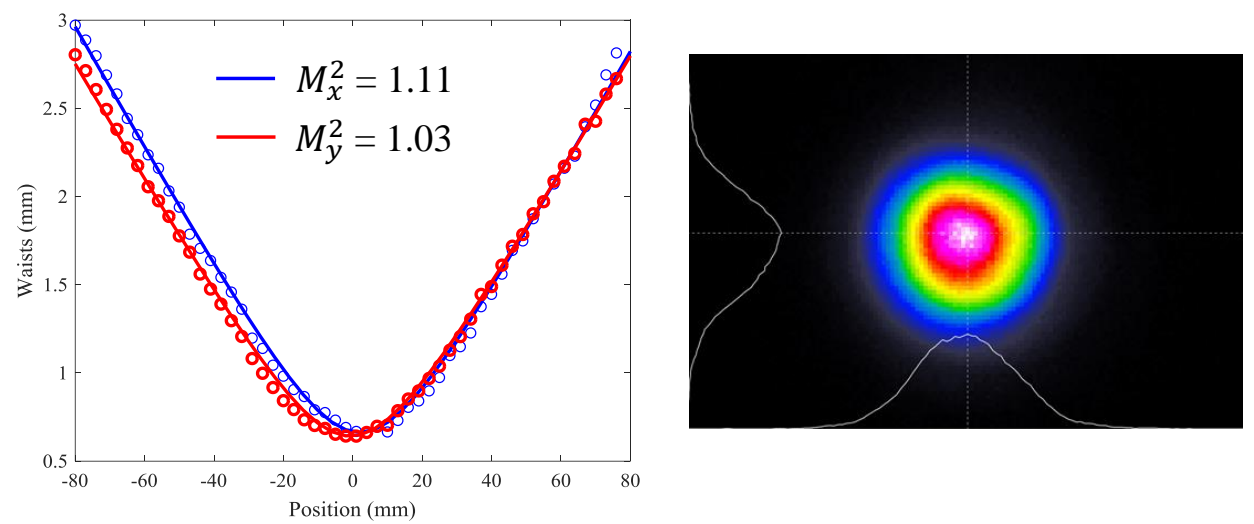

Fig. 7. Left: $\mathrm{M}^{2}$ measurement of the 61 combined beam before compression. Right: near field after central lobe selection (spatial filtering at the first dark ring).

Finally, the quality of the compressed pulse was evaluated at low power regime. The combined and spatially filtered beam was temporally compressed by using a $17601 / \mathrm{mm}$ multilayer dielectric reflection grating pair. The compressed pulse autocorrelation is depicted in Fig. 8, giving a FWHM duration of $362 \mathrm{fs}$, corresponding to a $257 \mathrm{fs}$ pulse duration assuming a Gaussian pulse. 


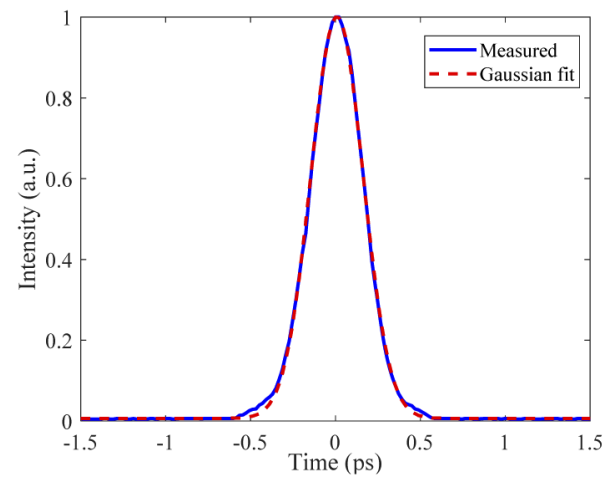

Fig. 8. Autocorrelation trace of the compressed output combined beam in linear regime.

\section{Discussion, conclusion and outlook}

As mentioned in section 1, the XCAN project aims at demonstrating a highly scalable CBC architecture in the femtosecond regime with 61 operated channels. When the 61 beams are power amplified to a pump-limited average power of $25 \mathrm{~W}$, the foreseen total average power is $600 \mathrm{~W}$. Considering the $\mathrm{CBC}$ and compressor efficiencies, the average power at the laser head output would be around $1.5 \mathrm{~kW}$. First results when operating the laser in such $\sim \mathrm{kW}$ average power regime at $2 \mathrm{MHz}$ repetition rate are given by Fig. 9, showing the far field evolution of the 61-combined beam over one- hour operation. The measured $\sim 1 \mathrm{~kW}$ average power and $500 \mu \mathrm{J}$ energy includes main and side lobes powers. At $t=0$, we measured a combining efficiency of $16 \%$. After an hour of laser operation, the $\mathrm{CBC}$ remains effective but the efficiency dropped to $12 \%$.
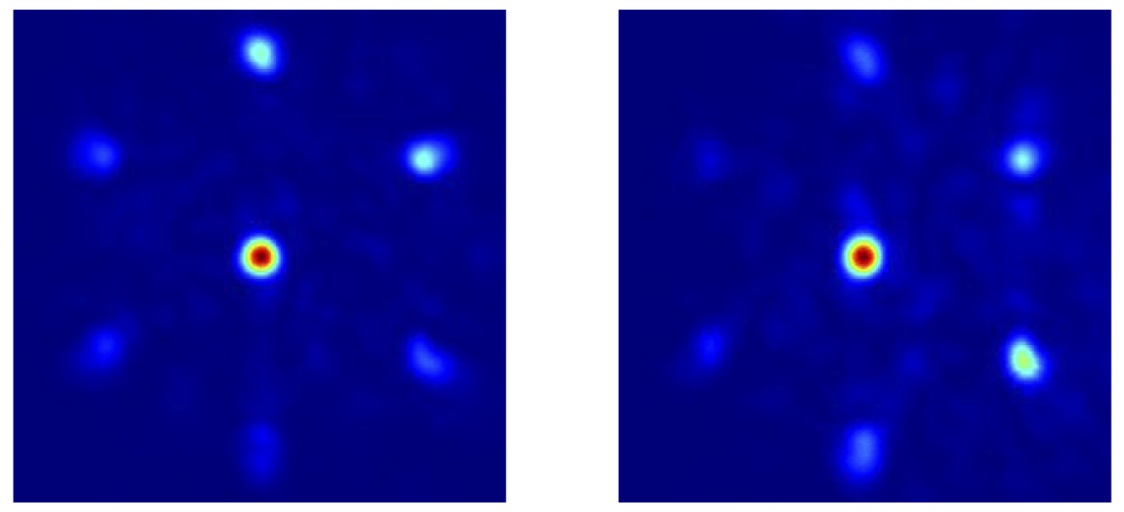

Fig. 9. Experimental far field of the $\mathrm{CBC}$ of 61 beams recorded at $\mathrm{t}=0$ (left) and at $\mathrm{t}=1 \mathrm{~h}$ (right) in the linear regime ( $2 \mathrm{MHz}$ repetition rate).

Near field interferometric analysis shows that the fringes contrast decreases for some fibers at a given positions within the 61 channel matrix. Simultaneously, we observe fringes rotation and curvature for some sub-pupils. Such interferometric pattern distortion is a signature of lateral and longitudinal mismatches between an individual beam and its dedicated collimating microlens.

These deteriorations are mainly related to thermal effects within the laser head, as no water cooling system is yet in place for all optomechanical elements. It is worth noting that, when re-operating the laser at low-to-moderate average power, these orientation, curvature and contrast issues vanish over a short time, as the thermal load on the laser head and the fiber amplifiers 
decreases permitting the recovering of a homogeneous and uniform near field interference pattern with high contrast and allowing the CBC efficiency to get back to more than 50\%.

To conclude, we have demonstrated the first coherent combination of 61 LMA fiber amplifiers in the femtosecond regime involving the tiled-aperture geometry along with an interferometric phase measurement technique. Such channel number sets a world record for coherently combined fiber amplifiers paving the way for a new generation of joule-class fiber lasers operating in the $\mathrm{kW}$ regime.

We believe that, by relying on the laser head architecture proposed in this work and by using a proper cooling system, adding more fibers is quite doable while respecting the $\mathrm{CBC}$ geometrical constraints. The individual phase control of each fiber is not an issue and our collective phase measurement approach shall manage up to 10000 fibers with the requested bandwidth.

One essential issue to be addressed at this stage of the XCAN project is the thermal effects management, a crucial task while operating the laser system in the $\mathrm{kW}$ average power regime. The next steps of this work will consist in of: (1) water cooling of all the laser head optomechanical elements and the fiber amplifiers breadboards; (2) operating the laser at various powers (with up to about half a $\mathrm{kW}$ of combined average power) and nonlinearity levels (B integral from 3 to 5 radians) and studying how these nonlinear effects might impact both temporal and spectral beam profiles or the coherent combination efficiency.

Finally, this laser prototype could be used as a programmable phase \& amplitude source to generate arbitrary shaped laser electric field distributions at a 61 "pixels" resolution level. Generation of high power Orbital Angular Momentum (OAM) beams as well as higher order non-symmetrical user-defined far field distributions is now under investigation.

\section{Funding}

French Ministry of Defense (Direction Générale de l'Armement); Horizon 2020 Framework Programme (654148 Laserlab-Europe); .

\section{Acknowledgments}

Authors would like to thank Optical Engines Inc. (USA) for fruitful discussions and collaboration. We are grateful to the LULI laboratory of the Ecole Polytechnique for hosting the XCAN project activities.

\section{Disclosures}

The authors declare no conflicts of interest.

\section{References}

1. T. Graf, R. Weber, M. Abdou-Ahmed, V. Onuseit, C. Freitag, M. Weidenmann, J-P. Negel, and A. Löscher, "Efficient High-Quality Processing of CFRP with a kW Ultrafast Thin-Disk Laser," in Advanced Solid State Lasers, OSA Technical Digest (online), paper AF2A.2 (2015).

2. S. Faas, R. Weber, and T. Graf, "Heat accumulation controlled surface functionalization of stainless steel with structuring rates up to $500 \mathrm{~mm}^{2} / \mathrm{s}$," Procedia CIRP 74, 324-327 (2018).

3. M. Jenne, D. Flamm, T. Ouaj, J. Hellstern, J. Kleiner, D. Grossmann, M. Koschig, M. Kaiser, M. Kumkar, and S. Nolte, "High-quality tailored-edge cleaving using aberration-corrected Bessel-like beams," Opt. Lett. 43(13), 3164-3167 (2018).

4. W. P. Leemans, "White paper of the ICFA-ICUIL joint task force: High power laser technology for accelerators," ICFA Beam Dyn. Newslett. 56, 10-88 (2011).

5. C. Saraceno, D. Sutter, T. Metzger, and M. Abdou Ahmed, "The amazing progress of high-power ultrafast thin-disk lasers,” J. Eur. Opt. Soc.-Rapid Publ. 15(1), 15 (2019).

6. T. Y. Fan, "Laser beam combining for high-power, high-radiance sources," IEEE J. Quantum Electron. 11(3), 567-577 (2005).

7. A. Brignon, (ed.) Coherent Laser Beam Combining, Wiley-VCH, (2013).

8. M. Antier, J. Bourderionnet, C. Larat, E. Lallier, E. Lenormand, J. Primot, and A. Brignon, "kHz closed loop interferometric technique for coherent fiber beam combining,” IEEE J. Quantum Electron. 20(5), 182-187 (2014). 


\section{Optics EXPRESS}

9. M. Müller, C. Aleshire, H. Stark, J. Buldt, A. Steinkopff, A. Klenke, A. Tünnermann, and J. Limpert, “10.4 kW coherently-combined ultrafast fiber laser," Proc. SPIE 11260, 10 (2020).

10. T. Zhou, T. Sano, and R. Wilcox, "Coherent combination of ultrashort pulse beams using two diffractive optics," Opt. Lett. 42(21), 4422-4425 (2017).

11. A. Klenke, M. Müller, H. Stark, A. Tünnermann, and J. Limpert, "«Sequential phase locking scheme for a filled aperture intensity coherent combination of beam arrays," Opt. Express 26(9), 12072 (2018).

12. W. Leemans, B. Nagler, A. J. Gonsalves, C. Toth, K. Nakamura, C. G. R. Geddes, E. Esarey, C. B. Schroeder, and S. M. Hooker, "GeV electron beams from a centimetre-scale accelerator," Nat. Phys. 2(10), 696-699 (2006).

13. G. Mourou, B. Brocklesby, T. Tajima, and J. Limpert, "The future is fibre accelerators," Nature 7(4), 258-261 (2013).

14. T. Ebisuzaki, M. Quinn, S. Wada, L. Piotrowski, Y. Takizawa, M. Casolino, M. Bertaina, P. Gorodetzky, E. Parizot, T. Tajima, R. Soulard, and G. Mourou, "Demonstration designs for the remediation of space debris from the International Space Station," Acta Astronaut. 112, 102-113 (2015).

15. M. N. Quinn, V. Jukna, T. Ebisuzaki, I. Dicaire, R. Soulard, L. Summerer, A. Couairon, and G. Mourou, "Space-based application of the CAN laser to LIDAR and orbital debris remediation,” Eur. Phys. J.: Spec. Top. 224(13), 2645-2655 (2015).

16. A. Heilmann, J. Le Dortz, L. Daniault, I. Fsaifes, S. Bellanger, J. Bourderionnet, C. Larat, E. Lallier, M. Antier, E. Durand, C. Simon-Boisson, A. Brignon, and J.-C. Chanteloup, "Coherent beam combining of seven fiber chirped-pulse amplifiers using an interferometric phase measurement," Opt. Express 26(24), 31542 (2018).

17. J. Le Dortz, A. Heilmann, M. Antier, J. Bourderionnet, C. Larat, I. Fsaifes, L. Daniault, S. Bellanger, C. Simon Boisson, J.-C. Chanteloup, E. Lallier, and A. Brignon, "Highly scalable femtosecond coherent beam combining demonstrated with 19 fibers," Opt. Lett. 42(10), 1887-1890 (2017).

18. I. Fsaifes, L. Daniault, A. Heilmann, J. Le Dortz, S. Bellanger, J. Bourderionnet, C. Larat, E. Lallier, M. Antier, E. Durand, A. Brignon, and J-C. Chanteloup, "Coherent Beam combining of 37 femtosecond fiber amplifiers," Conference on Lasers and Electro-Optics Europe, Munich, Germany. Paper CA-1.6, (2019).

19. J. Bourderionnet, C. Bellanger, J. Primot, and A. Brignon, "Collective coherent phase combining of 64 fibers," Opt. Express 19(18), 17053 (2011).

20. A. Heilmann, "Highly scalable femtosecond coherent beam combining system of high power fiber amplifiers" PhD Thesis, http://www.theses.fr/2018SACLX112.

21. M. A. Vorontsov and V. P. Sivokon, "Stochastic parallel-gradient-descent technique for high-resolution wave-front phase-distortion correction," J. Opt. Soc. Am. A 15(10), 2745-2758 (1998). 